\title{
Acoustic Transport of Electrons in Parallel Quantum Wires
}

\author{
J. Cunningham ${ }^{a, *}$, M. Pepper $^{b}$, V.I. TalyanskiI ${ }^{b}$ \\ AND D.A. RITCHIE ${ }^{b}$ \\ ${ }^{a}$ School of Electronic and Electrical Engineering, The University of Leeds \\ Woodhouse Lane, Leeds, LS2 9JT, UK \\ ${ }^{b}$ Cavendish Laboratory, Madingley Road, Cambridge, CB3 0HE, UK \\ Over the last few years we have developed a new method to control \\ single-electrons by isolating and moving them through a submicron width \\ channel formed in a GaAs/AlGaAs heterostructure using a surface acoustic \\ wave. The acoustic wave acts to push electrons through the depleted sub- \\ micron channel in packets each containing an integer number of electrons. \\ Our primary motivation for studying this system has been to develop a new \\ standard of dc current for metrological purposes, but our recent focus has \\ widened to investigate the possibility of single-photon emission. Here we \\ show new experimental results which demonstrate acoustoelectric current \\ flow in adjacent $1 \mathrm{D}$ wires. These results have relevance both to the use of \\ the system in a single-photon emission scheme, as well as in the creation of \\ a proposed acoustoelectric quantum computer.
}

PACS numbers: 73.21.Hb, 72.50.+b, 73.50.Rb, 77.65.Dq

\section{Introduction}

In the last few years we have developed a method to control single-electrons moving them through a submicron width channel formed in a GaAs/AlGaAs heterostructure using a $\mathrm{GHz}$ frequency surface acoustic wave (SAW) [1-6]. A highly accurate dc current composed of a train of single-electrons is generated by a SAW moving electrons through the depleted channel in packets that each contain an integer number of electrons. We have performed a number of experiments on this system to assess the accuracy and stability of the current generated [1-6]. Two proposals put forward to exploit this accurate single-electron current in practical devices are a single-photon source in which single-electrons are deposited in a $p$-type region to recombine [7], and a quantum computer where single-electrons trapped by the surface acoustic wave are used as qubits [8]. A central requirement of both these proposals is the control of acoustoelectric current in submicron

*corresponding author; e-mail: eenjec@leeds.ac.uk 
separated wires. Here we present a system that demonstrates such control as we measure the acoustoelectric current passing through two wires fabricated with submicron separation. This control is achieved through adjusting the acoustoelectric current in each channel with Schottky gates.

In the proposed single-photon emission scheme a flow of acoustoelectric current from a quantum wire is directed into a $p$-type region, where carrier recombination takes place, which leads to single-photon emission [7]. For accurate emission of single photons the recombination time in the $p$-type region, $\tau_{\mathrm{r}}$, must be much smaller than the time between the injection of consecutive single-electrons, $\tau_{\mathrm{i}}$. The fastest recombination time available in p-type GaAs is of order 100 ps, based on time resolved photoluminescence measurements of quantum wells using a streak camera [9], while $\tau_{\mathrm{i}}$ is set by the SAW wavelength. Successful measurements of acoustic single-electron transport have been performed at frequencies down to $\sim 1 \mathrm{GHz}$, or $\tau_{\mathrm{i}}=1 \mathrm{~ns}[10]$. Significantly longer wavelengths are impractical owing to the large impurity-free quasi-1D channel length required. The requirement that $\tau_{\mathrm{r}} \ll \tau_{\mathrm{i}}$ is therefore not well fulfilled by present SAW devices, where the two quantities are of similar order. Fast modulation of branched acoustoelectric current flow could provide a way to increase the time between subsequent electrons entering a $p$-type region. The single-electron current would be split into two paths using a fast-modulated gate or gates, yielding currents in the two paths equal to $I_{1}=x$ ef and $I_{2}=(x-1)$ ef, where $x$ is the proportion of time that path 1 is open [7]. Such a scheme is yet to be demonstrated however, and to be feasible the switching of acoustoelectric current between two closely spaced channels must first be achieved. Here we show experimental results that demonstrate separately modulated acoustoelectric current flow in adjacent wires in the open-channel regime. Our device allows us to measure the acoustoelectric current in each wire separately and simultaneously as the wires have a common source of electrons but separate collector regions. This contrasts with an earlier device which had shared source and collector [10] for two paths which were separated at their point of closest approach by 5 microns, the acoustoelectric current flowing in the two paths being added together before measurement.

\section{Device fabrication}

A schematic of the optical lithography mask design used for our device is shown in Fig. 1. The sample layout is similar to that used in previous investigations [5] with the exception of an additional Schottky gate to enable the formation of two branches for acoustoelectric current in the device. The structure shown was fabricated on a GaAs/AlGaAs high electron mobility transistor with a two-dimensional electron gas (2DEG) formed $90 \mathrm{~nm}$ below the surface. The dark carrier density of the wafer is $1.75 \times 10^{11} \mathrm{~cm}^{-2}$ and its mobility $0.89 \times 10^{6} \mathrm{~cm}^{2} \mathrm{~V}^{-1} \mathrm{~s}^{-1}$ measured at $4.2 \mathrm{~K}$. Figure 2 shows the electron beam lithography pattern defined on the 2DEG mesa. The Schottky contacts converge in this region near the centre 


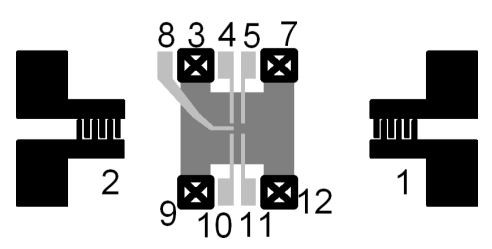

Fig. 1. Schematic diagram of the device. Surface acoustic waves can be launched from transducers 1 and 2. Annealed AuGeNi ohmic contacts are 3, 7, 9, and 12. Schottky gate contacts are $4,5,8,10$, and 11 . Contact 8 allows a gate to be fabricated after additional electron beam lithography which bisects the acoustoelectric current in the channel formed between contacts 4 and 10 (see Fig. 3). The dark grey area is the heterojunction mesa.
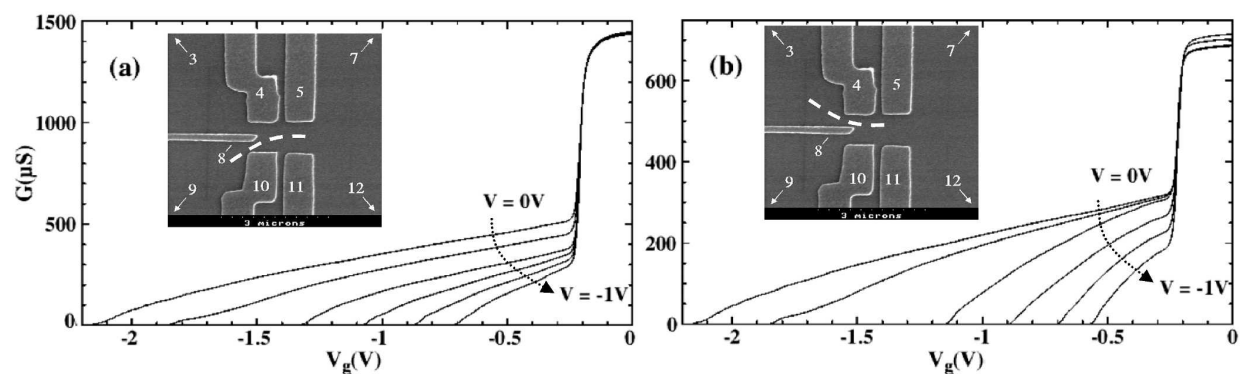

Fig. 2. Two-terminal conductance of the two channels formed between ohmic contacts (a) 9 and 12 and (b) 3 and 12. Finger gate 8 voltage was incremented between $0 \mathrm{~V}$ and $-1 \mathrm{~V}$ in $0.2 \mathrm{~V}$ steps as indicated. Series resistances comprising the ohmic contact and 2DEG resistance attached to the two channels are not deducted from the data. Insets: Electron micrograph of the Schottky gate pattern fabricated using electron beam lithography. Metallization is $\mathrm{NiCr} / \mathrm{Al} 20 \mathrm{~nm} / 40 \mathrm{~nm}$. Contacts referred to in the text are indicated. Dashed line represents the channel open to conduction in each case.

of the mesa, which is in the middle of the impinging surface acoustic wave beam (beam width $\sim 100 \mu \mathrm{m}$ ). A pair of opposing interdigital transducers for SAW production were fabricated using electron beam lithography on either side of the mesa with an interdigital pitch of $\sim 1 \mu \mathrm{m}$, yielding efficient SAW production near $2.7 \mathrm{GHz}$. The gate material was a $40 \mathrm{~nm}$ thick Al layer with $20 \mathrm{~nm} \mathrm{NiCr}$ adhesion layer, the electron-beam resist being a $180 \mathrm{~nm}$ thick layer of poly-(methyl methacrylate). A high contrast developer composed from a 20:1 mixture of A:B where $\mathrm{A}=$ MIBK:IPA in a volume ratio $1: 3$ and $\mathrm{B}=$ MEK:ethanol in a volume ratio 26.5:73.5 was used for both the transducer and gate lift-off.

\section{Results and discussion}

Experiments were performed on the device at $4.2 \mathrm{~K}$ in a $\mathrm{He}-4$ dewar with a purpose built microwave probe. SAW transducer efficiency was assessed using a HP8510 network analyzer. A minimum insertion loss of around $-65 \mathrm{~dB}$ 
near $2.7 \mathrm{GHz}$ for the opposing transducer pair indicates strongest SAW production for this frequency (Fig. 3a). The SAW was launched from transducer 1 (see Fig. 1b). Acoustoelectric current was measured between ohmic contacts 9 and 12, with a voltage applied to the split-gate formed by contacts 5 and 11 of $V_{\mathrm{g}}=-0.27 \mathrm{~V}$. This corresponded to the definition voltage of the constriction [1]. The result is seen in Fig. 3b. For transducer 2 acoustoelectric current flows in the opposite direction to transducer 1 , as expected, and the sign of the current for each transducer corresponds to electron transport. The observed difference in the level of acoustoelectric current for the two transducers could be due to small unintentional differences in impedance match between the transducers and the $50 \Omega$ co-axial feed line linking to room temperature, or the location of crystal defects relative to the transducers.

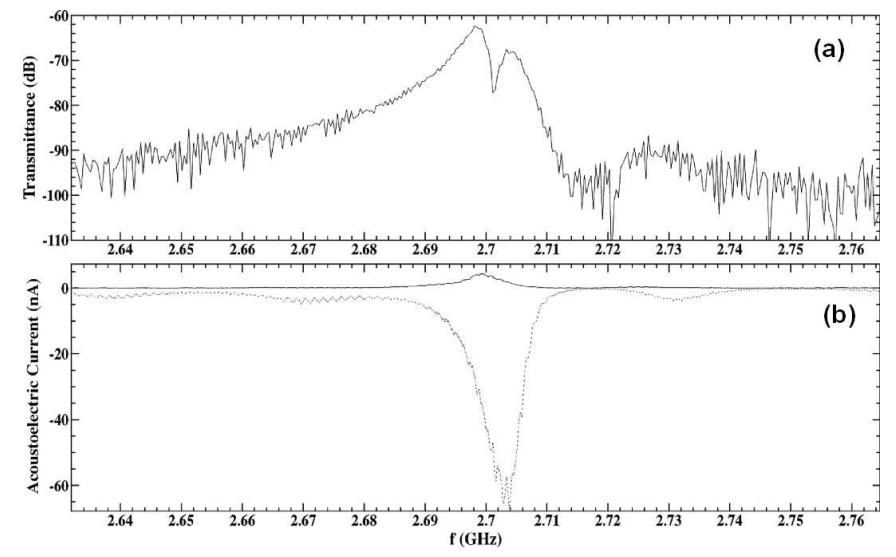

Fig. 3. (a) Transmittance of the delay line formed by the transducers on either side of the constriction $(T=4.2 \mathrm{~K})$. (b) Acoustoelectric current measured between ohmic contacts 3 and 12 at definition of gates 5 and $11\left(V_{\mathrm{g}}=-0.2 \mathrm{~V}\right)$ for an applied microwave power level of $+10 \mathrm{dBm}$. The upper (solid) trace is for transducer 1 , which launches SAWs from right to left of the figure, and the lower (dashed) trace is for transducer 2.

By applying a negative voltage to the split-gate formed between contacts 4 and 10, and the finger gate (contact 8), two submicron separated branches are defined in the 2DEG; one between ohmic contacts 9 and 12, the other between 3 and 12. For all the measurements described here the split-gate formed between contacts 5 and 11 was shorted to ground. Conductance traces were taken in a two-terminal, constant voltage $(100 \mu \mathrm{V})$ configuration between ohmic contacts 3 and 12, and 9 and 12. Figure 2 shows a series of traces taken for swept voltage applied to 4 and 10, with the finger gate (contact 8) voltage incremented as a parameter. As the finger gate is made more negative, the pinch-off voltages for the two channels move positive since the finger gate provides additional electrostatic confinement. The pinch-off voltage of the two parallel channels is different by $\sim 0.1 \mathrm{~V}$ for an 
applied finger gate voltage of $-1 \mathrm{~V}$. This small difference may be the action of an asymmetric ionized impurity potential in the vicinity of the channels acting to narrow one channel, or by a similar argument small unintentional asymmetries in the metallization of the Schottky gates.

We now explore the regime of device operation which demonstrates electrostatic control of the two channels. The static potential of the split-gate formed by Schottky contacts 4 and 10 is moved from side to side by applying different voltages to the two arms. Provided finger gate 8 is defined, the pinch-off voltage of the two channels then changes, and it is possible to select the path which is open to conduction. Conductance traces in Fig. 4 show this effect for a fixed voltage applied to the finger gate of $-0.45 \mathrm{~V}$. The top trace in Fig. $4 \mathrm{a}$ is taken with contact 4 biased starting at $+0.8 \mathrm{~V}$ relative to contact 10 . Both contacts are
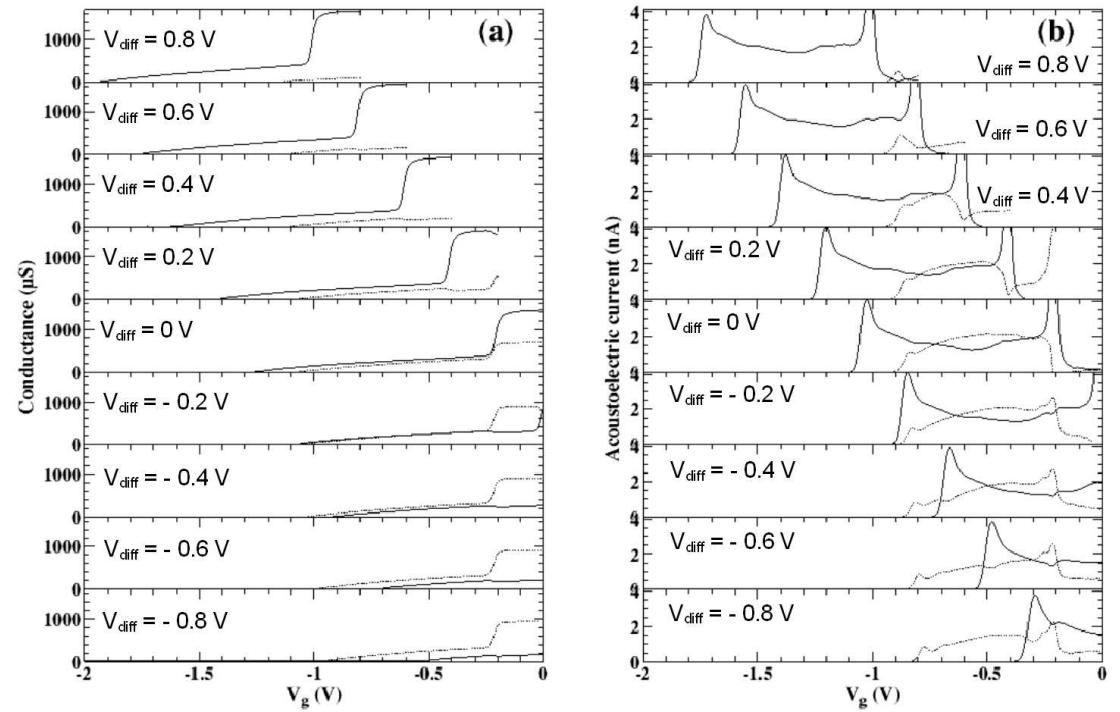

Fig. 4. (a) Two terminal conductance of the two channels formed by application of suitable negative potentials to the Schottky gates. Finger gate (contact 8) is at $-0.45 \mathrm{~V}$ and the voltage applied to contacts 4 and 10 is swept. All plots are shown as a function of the voltage applied to contact 4 . The difference in voltage between contacts 4 and 10 was an incremented parameter between panels, starting at $V_{\text {diff }}=0.8 \mathrm{~V}$ applied to contact 4 relative to $0 \mathrm{~V}$ on contact 10 in the top graph, moving down in $0.2 \mathrm{~V}$ increments to reach $0 \mathrm{~V}$ difference in the middle panel, and $-0.8 \mathrm{~V}$ on gate contact 10 in the bottom panel. Plots with continuous line are for the channel formed between ohmic contacts 9 and 12, while the dashed line is for the channel between ohmic contacts 3 and 12. (b) Acoustoelectric current measured for the two channels simultaneously using identical bias conditions to (a). SAWs were launched from the transducer 1 by applying a microwave signal of $+10 \mathrm{dBm}$ at $2.6991 \mathrm{GHz}$. 
then swept negative at the same rate thereby maintaining this relative difference of $0.8 \mathrm{~V}$ between them. For the upper panel in Fig. 4 the channel formed between ohmic contacts 3 and 12 pinches off well before that between 9 and 12 . This is because the electrostatic gap formed by contacts 4 and 8 is, for this choice of voltage difference between 4 and 10, narrower than the corresponding gap between contacts 10 and 8. Reducing the difference in voltage between the two arms in steps of $-0.4 \mathrm{~V}$ results in a series of traces which show channel 9 to 12 pinching off at increasingly positive gate voltage as the potential of gates is asymmetrically biased. Other traces in the same figure show the effect of continuing the shift in voltage to negative voltages applied on contact 10 relative to contact 4 with the pinch-off voltage of the two channels being nearly coincident at around $-0.1 \mathrm{~V}$ relative difference. The lower plots show that, for this range of voltages, channel 3 to 12 pinches off later than channel 9 to 12 .

Acoustoelectric current is induced in the two channels by launching a SAW from transducer 1 (Fig. 4b). For this series of measurements, transducer 1 was operated at its centre frequency of $2.6991 \mathrm{GHz}$ with an applied microwave power of $+15 \mathrm{dBm}$. The microwave power was pulse modulated using a $220 \mathrm{~Hz}$ square wave to act as a lock-in reference [1]. Measurements were again performed as a function of swept gate voltage with the difference in voltage between gate contacts 10 and 4 as an incremented parameter between sweeps. The finger gate (contact 8) was again held at $-0.45 \mathrm{~V}$. The action of differently biasing gate contacts 10 and 4 is again to asymmetrically adjust the two channel's static potential, resulting in different pinch-off voltages for acoustoelectric current in the two channels. Similar data to Figs. 2 and 4 were obtained at a second finger gate potential, $-0.65 \mathrm{~V}$, chosen to make the channels narrower. As expected both channels pinched off for a more positive gate voltage owing to this increased confinement, with traces otherwise similar.

Acoustoelectric current traces show a peak as a definition voltage of each quasi-1D channel, which can be attributed, in a similar way as the peak observed in single-channels, to the high value of the derivative of the 2DEG conductivity with respect to the carrier density at the definition voltage [1]. The acoustoelectric current in an open channel (the conducting regime) is very sensitive to impurity potentials, and both channels show some irregular features in the open channel regime which are a likely result of this. Similar behaviour was seen in early studies of single-channel devices [1]. This interpretation is also supported by the differing shapes of the acoustoelectric current seen between definition and pinch-off for the two channels and the slightly different pinch-off voltages of the two channels shown in Fig. 2. In the channel formed between contacts 9 and 12, small oscillations are seen in the open-channel regime between definition and pinch-off, which are likely to be a result of the subband structure which can be observed in cleaner channels [1]. As the pinch-off voltage is approached, the acoustoelectric current decreases until the channel is completely closed. 
We note that in Ref. [9] the authors showed the addition of quantized acoustoelectric current from two etched constrictions separated by approximately $5 \mu \mathrm{m}$, with the motivation of increasing the single-electron acoustoelectric current for metrological purposes. Our device is different since the electron flow in our channels is deposited into separate drains which are then independently measured. This difference enables our device to separate the acoustoelectric current generated in the two channels, and to independently control and measure this quantity.

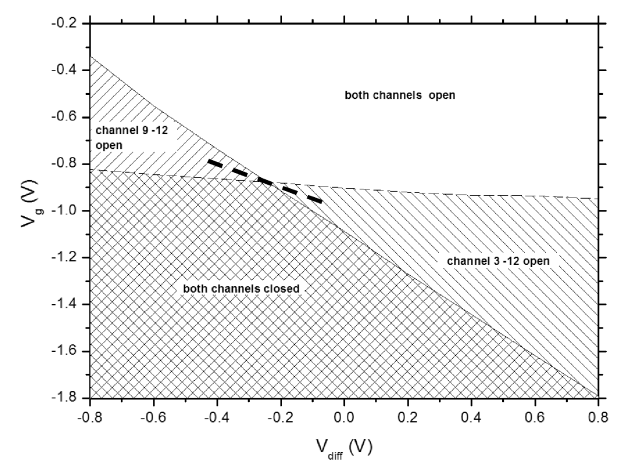

Fig. 5. Pinch-off voltages, defined as the points at which the acoustoelectric current moves below $5 \mathrm{pA}$, extracted from the shown data in Fig. 4b. Thin dashed line is for channel between ohmic contacts 9 and 12 and solid line for channel between ohmic contacts 3 and 12. Areas where one or the other channel conducts, where both conduct or where both are pinched off, are indicated by hatching. Thick dashed line indicates a voltage sweep which acts to open the channels 3 and 12, then 9 and 12 sequentially.

Figure 5 shows a summary of the pinch-off voltage data from Fig. 4 for the two channels. We see regimes of voltage where both channels are open, one or the other channel is open and both channels are closed. By applying suitable differential voltages we may follow a trajectory which will act to open one or the other path for acoustoelectric transport (such a trajectory is shown by the thick dashed line).

\section{Conclusion}

For devices of the type studied here to be useful in the single-photon emission scheme it must be demonstrated that the quantized acoustoelectric current is also separately controllable in the two branches. Further it will be necessary to demonstrate that the quantized current is switchable in the sense that the total magnitude of acoustoelectric current flowing in the two branches must remain constant, and that the speed of switching is sufficiently fast to enable accurate deflection of the electrons between two paths. These regimes of device operation are yet to be explored. 
Our results are also of relevance to a proposed acoustic quantum computer [8], whose aim is to perform two-qubit operations using the exchange coupling between single-electrons held within the acoustic minima in closely separated channels. Our measurements indicate that separate control and measurement of acoustoelectric current in channels fabricated in sub-micron proximity to one another is possible, and that complex arrays of Schottky gates such as those required by the proposed quantum computer could be used for this purpose.

In conclusion, we have studied acoustoelectric current flow in two submicron separated quantum wires. Using a suitable array of Schottky gates we have demonstrated control over the magnitude of acoustoelectric current flowing in each wire. The work represents progress towards implementations of both an acoustoelectric single-photon source and an acoustoelectric quantum computer.

\section{Acknowledgment}

This work was funded by the EPSRC and the EU, Project No. IST-2000-26020 (SAWPHOTON). J. Cunningham acknowledges support from the EPSRC for an Advanced Fellowship, and from the European Commission project "Center of Excellence in Processing, Research and Application of Advanced Materials" (PRAMA) for assistance in attending the symposium.

\section{References}

[1] J.M. Shilton, V.I. Talyanskii, M. Pepper, D.A. Ritchie, J.E.F. Frost, C.J.B. Ford, C.G. Smith, G.A.C. Jones, J. Phys. C 8, L531 (1996).

[2] V.I. Talyanskii, J.M. Shilton, M. Pepper, C.G. Smith, C.J.B. Ford, E.H. Linfield, D.A. Ritchie, G.A.C. Jones, Phys. Rev. B 56, 15180 (1997).

[3] V.I. Talyanskii, J.M. Shilton, J. Cunningham, M. Pepper, C.J.B. Ford, C.G. Smith, E.H. Linfield, D.A. Ritchie, G.A.C. Jones, Physica B 251, 140 (1998).

[4] J. Cunningham, V.I. Talyanskii, J.M. Shilton, M. Pepper, A. Kristensen, P.E. Lindelof, Phys. Rev. B 62, 1564 (2000).

[5] J. Cunningham, V.I. Talyanskii, J.M. Shilton, M. Pepper, M.Y. Simmons, D.A. Ritchie, Phys. Rev. B 60, 4850 (1999).

[6] A. Robinson, V.I. Talyanskii, M. Pepper, J. Cunningham, E.H. Linfield, Phys. Rev. B 65, 045313 (2002).

[7] C.L. Foden, V.I. Talyanskii, G.J. Milburn, M.L. Leadbeater, M. Pepper, Phys. Rev. A 62, 011803 (2000).

[8] C.H.W. Barnes, J.M. Shilton, A.M. Robinson, Phys. Rev. B 62, 8410 (2000).

[9] M. Cecchini, V. Piazza, F. Beltram, M. Lazzarino, M.B. Ward, A.J. Shields, H.E. Beere, D.A. Ritchie, Appl. Phys. Lett. 82, 636 (2003).

[10] J. Ebbecke, G. Bastian, M. Blocker, K. Pierz, F.J. Ahlers, Appl. Phys. Lett. 77, 2601 (2000). 